This completes the proof of the theorem.

Note by the editor: The referee points out that the result of Fuchs to which the author refers appears in a slightly more general form in E. Hille's book, Functional analysis and semi-groups, Amer. Math. Soc. Colloq. Publ. Vol. 31, Amer. Math. Soc., Providence, R. I., 1948, p. 487.

\title{
REFERENCES
}

1. L. Fuchs, A remark on the Jacobson radical, Acta Sci. Math. (Szeged) 14 (1952), 167-168.

2. M. Hall, The theory of groups, Macmillan, New York, 1959.

3. N. Jacobson, The radical and semi-simplicity for arbitrary rings, Amer. J. Math. 67 (1945), 300-320.

4. - Structure of rings, Amer. Math. Soc. Colloq. Publ. Vol. 37, Amer. Math. Soc., Providence, R. I., 1956.

Kossuth Lajos University of Debrecen and

Martin Luther University of HaLle-Wittenberg

\section{NOTE ON AN EMBEDDING THEOREM OF ADYAN}

\author{
GEORGE C. BUSH ${ }^{1}$
}

1. Introduction. Adyan [1] studied the problem of embedding a finitely presented semigroup in a group. He obtained a sufficient condition for the embeddability but his paper does not discuss the question of necessity. In this note we give a simple counter-example that shows the condition is not necessary.

2. Adyan's embedding theorem. Let $S$ be a finitely presented semigroup, in particular the semigroup with generators $a_{1}, a_{2}, \cdots, a_{n}$ and defining relations

$$
A_{k}=B_{k} ; \quad k=1,2, \cdots, m
$$

where $A_{k}, B_{k}$ are products of positive powers of the generators and no $A_{k}, B_{k}$ is empty.

With each defining relation $A_{k}=B_{k}$ associate an unordered pair $\left(a_{i_{k}}, a_{j_{k}}\right)$ where $A_{k}$ has $a_{i_{k}}$ as its first generator and $B_{k}$ has $a_{j_{k}}$ or vice versa. This unordered pair is called the left pair of the defining relation $A_{k}=B_{k}$. The right pair is defined similarly, using the last generator from $A_{k}$ and from $B_{k}$.

Received by the editors May 14, 1962.

${ }^{1}$ This note was prepared while the author held a studentship of the National Research Council of Canada. 
By the left graph of the system (1) we mean the graph determined by the set of all generators that occur in (1) and the set of all left pairs of these relations. We say that the system (1) has no left cycles if the corresponding left graph contains no cycles. The right graph and right cycles can be defined similarly. If the system (1) has no left or right cycles it is said to contain no cycles.

Adyan's embedding theorem is as follows:

THEOREM (ADYAN). If the system of defining relations (1) of a semigroup $S$ has no cycles, then $S$ is isomorphic to a subsemigroup of the group $G$ with defining relations (1).

3. A counter-example. That the absence of cycles is not a necessary condition for embeddability is shown by the following example.

Let $S$ be the semigroup with generators $a_{1}, a_{2}, a_{3}, x_{1}, x_{2}$ and defining relations

$$
\begin{aligned}
& a_{1} x_{1}=a_{2} x_{2} \\
& a_{2} x_{1}=a_{3} x_{2} \\
& a_{3} x_{1}=a_{1} x_{2} .
\end{aligned}
$$

The system (2) clearly has a left cycle. We shall show that it is embeddable. In order to do this we make use of some results of Mal'cev $[2 ; 3]$.

An equation $A=B$ involving only non-negative powers of the generators of $S$ is called a group consequence of $S$ if it holds in the group whose defining relations are (2). Consider a set of group consequences of $S$. We shall denote such a set by $C(S)$ for convenience, although this is not unique for $S$. Let $S^{\prime}$ be the semigroup with the equations of $C(S)$ together with (2) as defining relations. If every group consequence of $S^{\prime}$ is also true in $S^{\prime}$ we say that $C(S)$ together with (2) is a complete set of group consequences of $S$.

An application of one of Mal'cev's lemmas [3, Lemma 2] shows that the set of all group consequences of $S$ of the forms $a_{i} x_{j}=a_{k} x_{m}, a_{i}=a_{j}$, $x_{i}=x_{j}$ is a complete set of group consequences. We shall show that the only equations of this form that are group consequences are the equations (2) and identities.

We shall first show that the only equations $a_{i}=a_{j}$ or $x_{i}=x_{j}$ that are group consequences are identities. To do this we need only exhibit a group for which equations (2) hold and for which the $a_{i}$ 's are distinct and $x_{1} \neq x_{2}$.

Consider $Z /(7)$, the multiplicative group of nonzero residue classes 
of integers modulo 7 . If we take $a_{1} \equiv 1, a_{2} \equiv 4, a_{3} \equiv 2, x_{1} \equiv 1, x_{2} \equiv 2$ we can verify that equations (2) hold.

Now consider the nonidentical equations of the form $a_{i} x_{j}=a_{k} x_{m}$ other than the equations (2). Of these an equation of the form $a_{i} x_{j}$ $=a_{i} x_{k}$ cannot be a group consequence, for then $x_{j}=x_{k}$, contrary to what we have just proved. Similarly $a_{i} x_{j}=a_{k} x_{j}$ cannot be a group consequence. The only equations that remain to be considered are $a_{1} x_{1}=a_{3} x_{2}, a_{1} x_{2}=a_{2} x_{1}$, and $a_{2} x_{2}=a_{3} x_{1}$. If $a_{1} x_{1}=a_{3} x_{2}$ is a group consequence then, since $a_{1} x_{1}=a_{2} x_{2}$ we have $a_{2} x_{2}=a_{3} x_{2}$ in the group, which contradicts $a_{2} \neq a_{3}$. Thus $a_{1} x_{1}=a_{3} x_{2}$ is not a group consequence. Similarly it can be shown that $a_{1} x_{2}=a_{2} x_{1}$ and $a_{2} x_{2}=a_{3} x_{1}$ are not group consequences.

This completes the proof that (2) is a complete set of group consequences of $S$ and hence that all the group consequences of $S$ already hold in $S$. The embedding theorem of [2] as restated in [3] now shows that $S$ can be embedded in a group.

We now have a semigroup $S$ with a left cycle which is embeddable in a group. Thus the absence of cycles cannot be a necessary condition for embeddability.

\section{REFERENCES}

1. S. I. Adyan, On the embeddability of semigroups in groups, Soviet Math. 1 (1960), 819-821.

2. A. Mal'cev, Über die Einbettung von assoziativen Systemen in Gruppen, Mat. Sb. (N.S.) 6(48) (1939), 331-336. (Russian, German summary)

3. - Über die Einbettung von assoziativen Systemen in Gruppen. II, Mat. Sb. (N.S.) 8(50) (1940), 251-264. (Russian, German summary)

QUEEN'S UNIVERSITY 